TRANSACTIONS OF THE

AMERICAN MATHEMATICAL SOCIETY

Volume 353, Number 8, Pages 3229-3246

S 0002-9947(01)02555-7

Article electronically published on April 9, 2001

\title{
LINK COMPLEMENTS AND THE BIANCHI MODULAR GROUPS
}

\author{
MARK D. BAKER
}

\begin{abstract}
We determine the values of $m$ for which the Bianchi modular group $\mathrm{PSL}_{2}\left(\mathcal{O}_{m}\right)$ contains a link group.
\end{abstract}

\section{INTRODUCTION AND STATEMENT OF RESUlTS}

Let $\mathcal{O}_{m}$ be the integers of the imaginary quadratic number field $\mathbb{Q}(\sqrt{-m})$. The Bianchi modular group, $\operatorname{PSL}_{2}\left(\mathcal{O}_{m}\right)$, is a discrete subgroup of $\mathrm{PSL}_{2}(\mathbb{C})$ the orientation preserving isometries of hyperbolic 3 -space, $\mathbb{H}^{3}$.

In this paper we determine those values of $m$ for which $\operatorname{PSL}_{2}\left(\mathcal{O}_{m}\right)$ contains a link group, that is, a torsion-free subgroup of finite index, $\Gamma$, such that $\mathbb{H}^{3} / \Gamma$ is homeomorphic to a link complement in $S^{3}$.

Results on the cohomology of $\mathrm{PSL}_{2}\left(\mathcal{O}_{m}\right)$ (see $\left.\mathrm{V}\right]$ ) limit the values of $m$ for which this is possible to the following list, $\mathcal{L}$ :

$$
\mathcal{L}=\{1,2,3,5,6,7,11,15,19,23,31,39,47,71\} .
$$

Over the last 20 years, numerous link groups have been found in $\operatorname{PSL}_{2}\left(\mathcal{O}_{m}\right)$ for the seven cases $m=1,2,3,7,11,15,23$ (see [B], [H1], [R1, [T], Wi]). Furthermore, many of the corresponding links (figure eight knot, Whitehead link, Borromean rings,...) have been central to the study of 3-manifolds.

We prove that the seven remaining Bianchi groups from the above list, $\mathcal{L}$, also contain link groups; hence:

Theorem. The Bianchi group $\mathrm{PSL}_{2}\left(\mathcal{O}_{m}\right)$ contains a link group if and only if $m \in \mathcal{L}$.

\section{Preliminaries and outline of proof}

Henceforth, let $\Omega_{m}$ denote the orbifold $\mathbb{H}^{3} / \mathrm{PSL}_{2}\left(\mathcal{O}_{m}\right)$. We prove our result by:

i) Embedding $\Omega_{m}$ in $S^{3}$ as the complement of a link of circles. The orbifolds $\Omega_{m}$ for $m=5,6,15,19,23,31,39,47,71$ are drawn in Figure 1.

ii) Using the embeddings in (i) to prove that $\Omega_{m}$ admits a finite sheeted orbifold covering $\widetilde{\Omega}_{m} \rightarrow \Omega_{m}$ such that $\widetilde{\Omega}_{m}$ is a nonsingular hyperbolic link complement.

Thus $\widetilde{\Omega}_{m} \cong \mathbb{H}^{3} / \Gamma$, where $\Gamma$ is a torsion-free finite index subgroup of $\operatorname{PSL}_{2}\left(\mathcal{O}_{m}\right)$ and hence $\Gamma$ is a link group (see $\left[T\right.$, Chapter 13). The existence of $\widetilde{\Omega}_{m}$ follows from an analysis of the singular locus of $\Omega_{m}$.

In Section [3] we construct the link complements, $\widetilde{\Omega}_{m}$. We also apply our methods to construct link complements corresponding to subgroups of $\operatorname{PSL}_{2}\left(\mathcal{O}_{15}\right)$ and

Received by the editors June 3, 1998 and, in revised form, March 8, 1999.

2000 Mathematics Subject Classification. Primary 57M25; Secondary 11F06.

Key words and phrases. Link complements, Bianchi modular groups. 

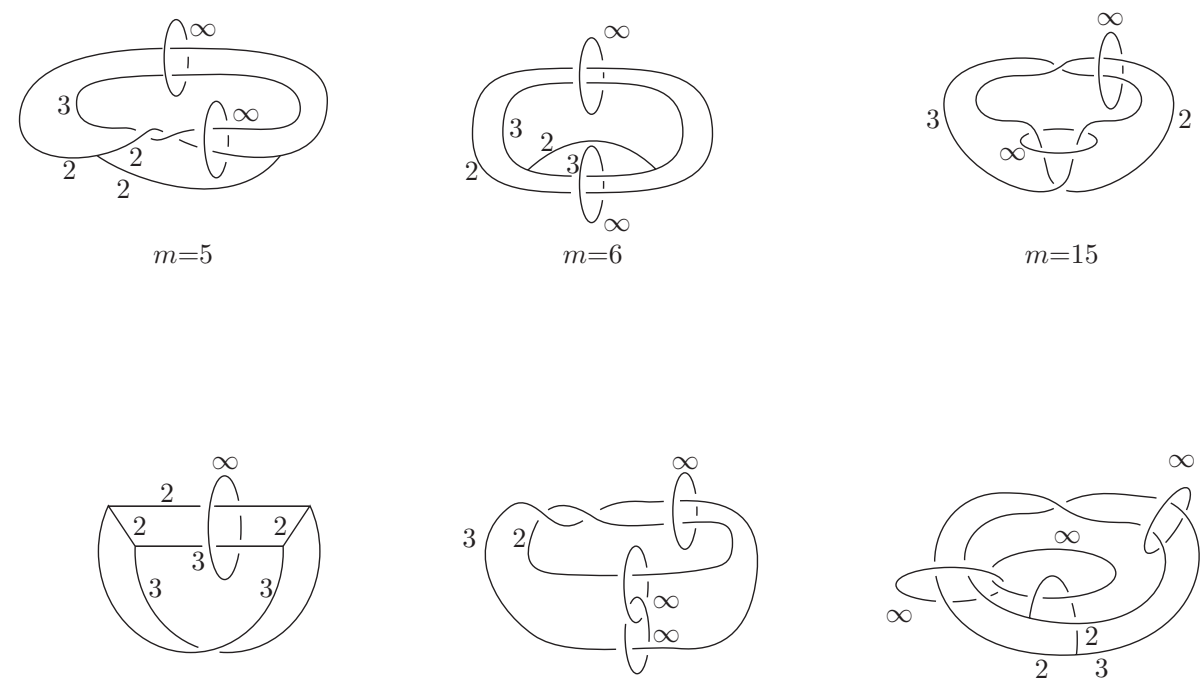

$m=19$

$m=23$

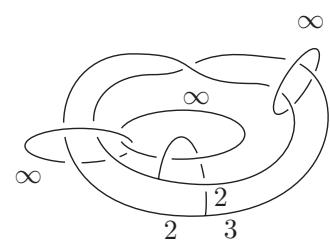

$m=31$
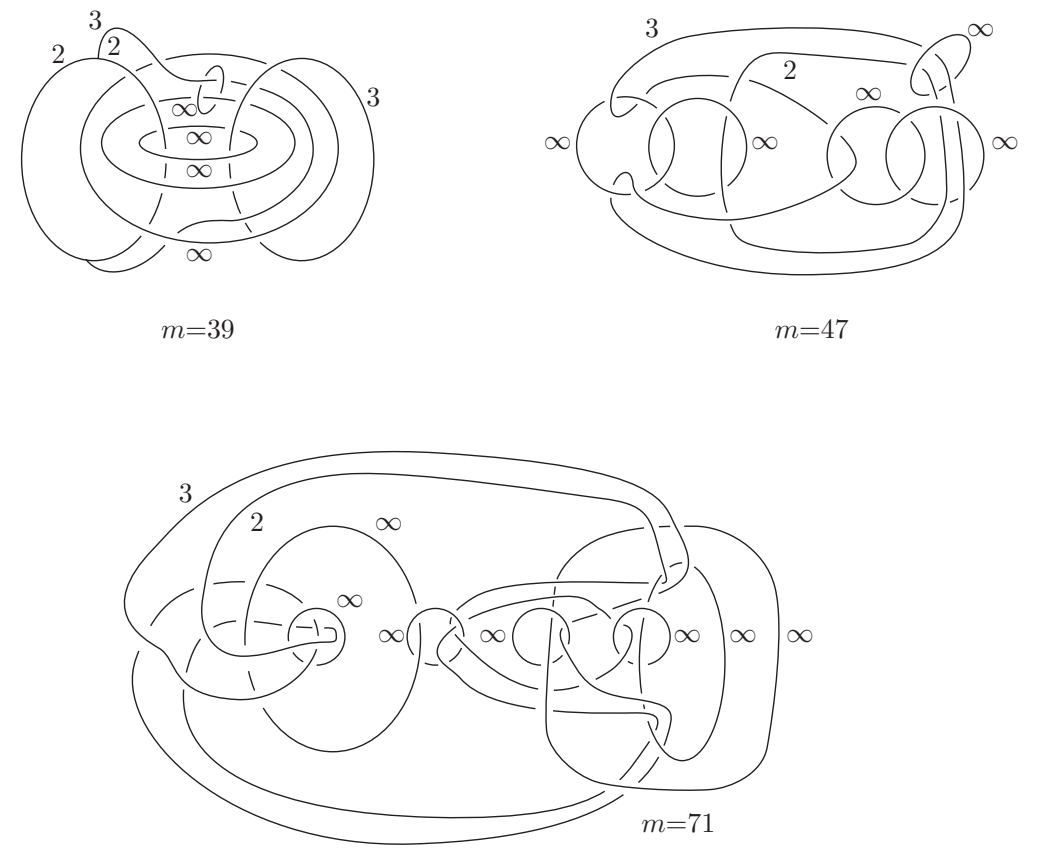

FiguRE 1. The orbifolds $\Omega_{m}$

$\mathrm{PSL}_{2}\left(\mathcal{O}_{23}\right)$ giving further examples in these two cases (see $\mathrm{B}$ for previous examples). Section 4 is devoted to a discussion of the orbifolds $\Omega_{m}$ and their embeddings in $S^{3}$. In Section 5 we give the volumes of $\widetilde{\Omega}_{m}$ calculated using SnapPea. Comparing these volumes with expected theoretical values provides a good check of our results. 


\section{Link COMPlements: The COVERs $\widetilde{\Omega}_{m}$}

Consider the orbifolds $\Omega_{m}$ drawn in Figure 1, Each $\Omega_{m}$ is a singular link complement. The components of the link are labelled by $\infty$, while segments of the singular locus of cone angle $\pi$ (resp. $2 \pi / 3$ ) are labelled by 2 (resp. 3 ).

3.1. The cases $\Omega_{m}, m=15,23,47,71$. Notice that the singular locus of these four orbifolds consists of two unknotted circles - one of cone angle $\pi$, the other of cone angle $2 \pi / 3$ - linked as shown:

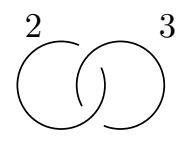

Now we simply use the fact that the $n$-fold cyclic cover of $S^{3}$ branched over an unknotted circle is homeomorphic to $S^{3}$. Let $\Omega_{m}^{\prime} \rightarrow \Omega_{m}$ be the 3 -fold cyclic cover branched over the circle of cone angle $2 \pi / 3$. Then the orbifold $\Omega_{m}^{\prime}$ is a link complement with singular locus an unknotted circle $\Sigma$ of cone angle $\pi$. Hence the 2 -fold cover $\widetilde{\Omega}_{m} \rightarrow \Omega_{m}^{\prime}$ branched over $\Sigma$ gives the desired nonsingular link complement.

We have drawn these links for $m=15,23,47$ in Figures 20 As for $m=71$, the complexity of the resulting link is such that we leave this as an exercise for the reader!

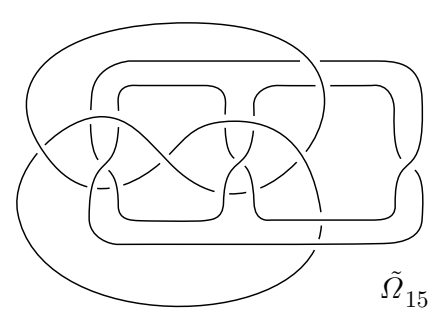

FIGURE 2.

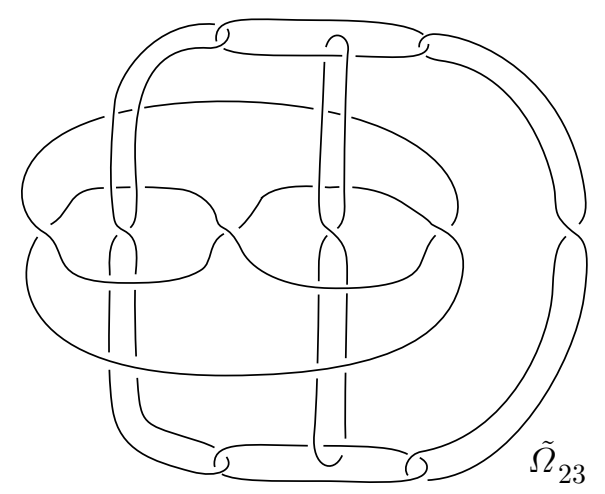

FiguRE 3. 


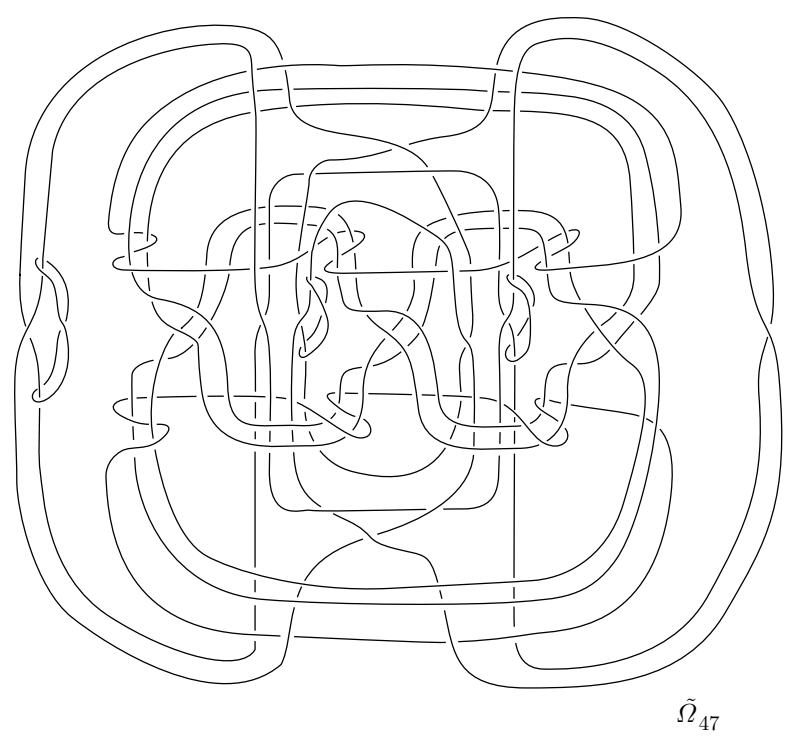

FiguRE 4.

3.2. The case $\Omega_{31}$. The singular locus of $\Omega_{31}$ is:

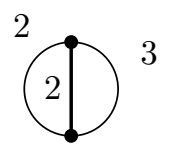

The edges of cone angle $\pi$ form an unknotted circle, $\Sigma_{2}$. Let $\Omega_{31}^{\prime} \rightarrow \Omega_{31}$ be the 2 -fold cyclic cover, branched over $\Sigma_{2}$. Thus the orbifold $\Omega_{31}^{\prime}$, is again a link complement, with singular locus an unknotted circle, $\Sigma_{3}$, of cone angle $2 \pi / 3$. Hence the 3 -fold cyclic cover of $\Omega_{31}^{\prime}$ branched over $\Sigma_{3}$ is a nonsingular link complement, drawn in Figure 5 .

3.3. The cases $\Omega_{m}, m=5,6,39$. We construct the covers $\widetilde{\Omega}_{m} \rightarrow \Omega_{m}$ using, in addition to the above techniques, the following lemma.

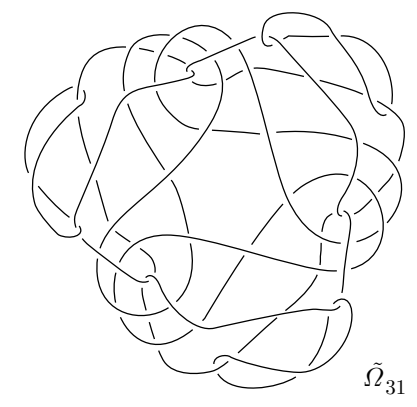

FIGURE 5 . 


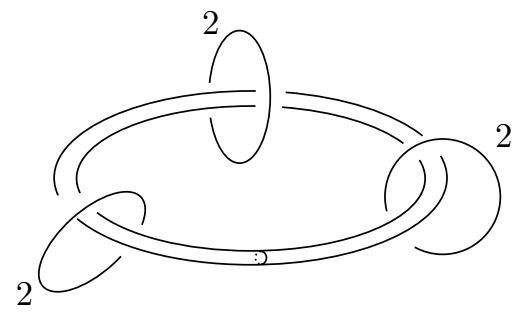

FiguRe 6a.

Lemma. (a) The orbifold $\mathrm{C}_{2}^{\infty} \bigcap_{2}$ is 2-fold branch covered by $\overbrace{\infty}^{\infty}$.

(b) The orbifold ${ }_{2}\left(\bigcap_{3}\right.$ is 3-fold (irregular) branch covered by ${ }_{2} \bigcap_{\infty}^{\infty}$.

Proof. We prove (b). The proof of (a) is analogous. We begin by compactifying the orbifold (b) by removing a tubular neighborhood of its link. Now consider its 3-fold cyclic cover, branched over $\Sigma_{3}$ pictured in Figure 6a. The preimage of $\Sigma_{2}$ consists of three unknotted circles of cone angle $\pi$. We further identify two of these singular circles by cutting along their (punctured) bounding disks and then identifying $D_{1}^{+}$with $D_{2}^{-}$(resp. $D_{1}^{-}$with $D_{2}^{+}$) by pushing everything through the tube, $T$ (Figures 6b 6c).

Notice that in these three cases, $\Omega_{m}$ contains the configuration of lemma (b):

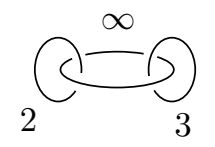

Let $\Omega_{m}^{\prime} \rightarrow \Omega_{m}$ be the corresponding 3-fold irregular branched cover.

3.3.1. The case $\Omega_{5}$. The construction of $\Omega_{5}^{\prime}$ is illustrated in Figure $7 \mathrm{a}$, The singular locus of $\Omega_{5}^{\prime}$ is ${ }^{2}{ }^{2}$ which we desingularize by taking cyclic covers as illustrated in Figure 7b, We obtain $\widetilde{\Omega}_{5}$ an 8-circle nonsingular link complement.

3.3.2. The case $\Omega_{6}$. The 3 -fold irregular cover $\Omega_{6}^{\prime} \rightarrow \Omega_{6}$ is drawn in Figure $8 \mathrm{a}$, Now $\Omega_{6}^{\prime}$ contains the configuration of lemma (a); hence we obtain the 2 -fold branched cover $\Omega_{6}^{\prime \prime} \rightarrow \Omega_{6}^{\prime}$ pictured in Figure $8 \mathrm{~b}$, The singular locus of $\Omega_{6}^{\prime \prime}$ is one circle of cone angle $\pi$, so that its 2 -fold branched cover yields the 12 -circle link complement in Figure $8 \mathrm{c}$

3.3.3. The case $\Omega_{39}$. The 3 -fold irregular branched cover $\Omega_{39}^{\prime} \rightarrow \Omega_{39}$ is drawn in Figure 9, The singular locus of $\Omega_{39}^{\prime}$ is:

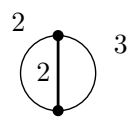

Thus (by applying verbatim the steps in 93.2 ) we know that $\Omega_{39}^{\prime}$ is 6 -fold covered by a nonsingular link complement. Given the complexity of this link, we do not draw it here. 


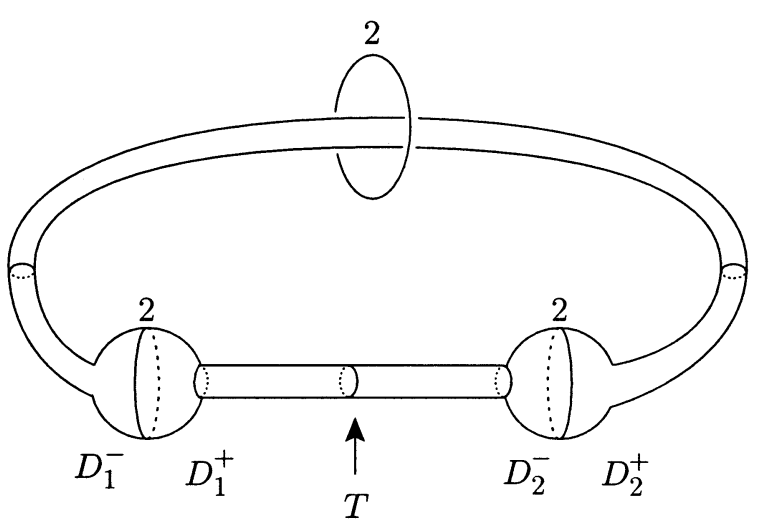

FiguRE $6 \mathrm{~b}$.
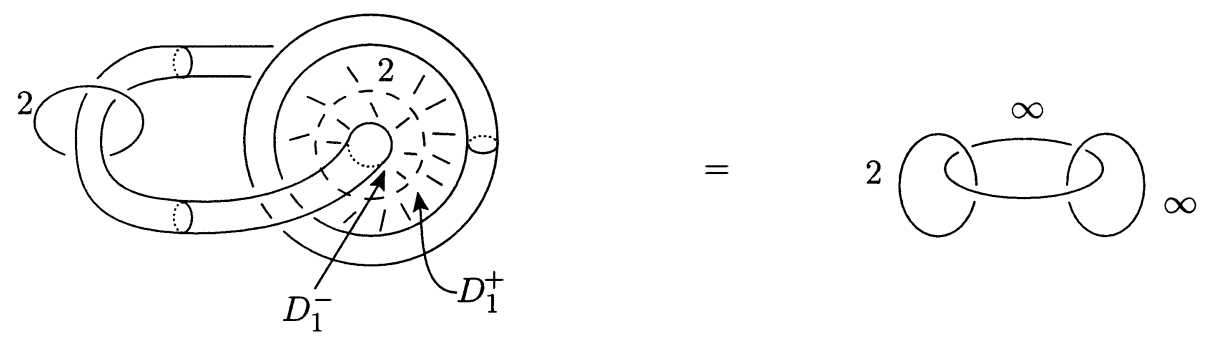

Figure 6c.

3.4. The case $\Omega_{19}$. The orbifold $\Omega_{19}$, owing to its complicated singular locus, was the hardest to deal with. We began by considering the quotient group

$$
\operatorname{PSL}_{2}\left(\mathcal{O}_{19}\right) /\left\langle\left\langle\left[\begin{array}{cc}
1 & \omega \\
0 & 1
\end{array}\right]\right\rangle\right\rangle, \quad \omega=\frac{-1+\sqrt{-19}}{2},
$$

which the computer program GAP $([\bar{G}])$ found to be of order 60 . This implies the following:

Proposition. The congruence subgroup $\Gamma(I) \subset \mathrm{PSL}_{2}\left(\mathcal{O}_{19}\right), I=(\omega)$, is the fundamental group of a link complement in a homotopy 3-sphere.

Proof. Recall that $\Gamma(I)$ is the kernel of $\operatorname{PSL}_{2}\left(\mathcal{O}_{19}\right) \rightarrow \operatorname{PSL}_{2}\left(\mathcal{O}_{19} / I\right)$ (see $[\mathrm{S}$ ] for details). Since $\mathcal{O}_{19} / I \cong \mathbb{Z} / 5 \mathbb{Z}$, it follows that $\Gamma(I)$ is a normal, torsion-free subgroup of index 60 in $\operatorname{PSL}_{2}\left(\mathcal{O}_{19}\right)$ containing the matrix $\left[\begin{array}{ll}1 & \omega \\ 0 & 1\end{array}\right]$. Thus $\Gamma(I)=\left\langle\left\langle\left[\begin{array}{ll}1 & \omega \\ 0 & 1\end{array}\right]\right\rangle\right\rangle$ so that $\Gamma(I)$ is generated by $\operatorname{PSL}_{2}\left(\mathcal{O}_{19}\right)$-conjugates of the parabolic element $\left[\begin{array}{ll}1 & \omega \\ 0 & 1\end{array}\right]$. Hence one can perform Dehn filling on the ends of $\mathbb{H}^{3} / \Gamma(I)$ so as to obtain a simply connected 3-manifold.

We will show that $\mathbb{H}^{3} / \Gamma(I)$ is in fact a link complement in $S^{3}$ (which we do not draw) as well as give explicitly a 4-component link complement $\widetilde{\Omega}_{19}$ (Figure 10c), that is a 12 -sheeted orbifold cover of $\Omega_{19}$.

Let $X$ denote $\Omega_{19}$ with its link circle put back (Dehn fill $\Omega_{19}$ with respect to the meridian $\left.\left[\begin{array}{ll}1 & \omega \\ 0 & 1\end{array}\right]\right)$. Note that $X$ is topologically $S^{3}$. Both of the above results follow from the fact that $X$ admits a 6 -fold irregular orbifold cover, $X^{\prime}$, that is 

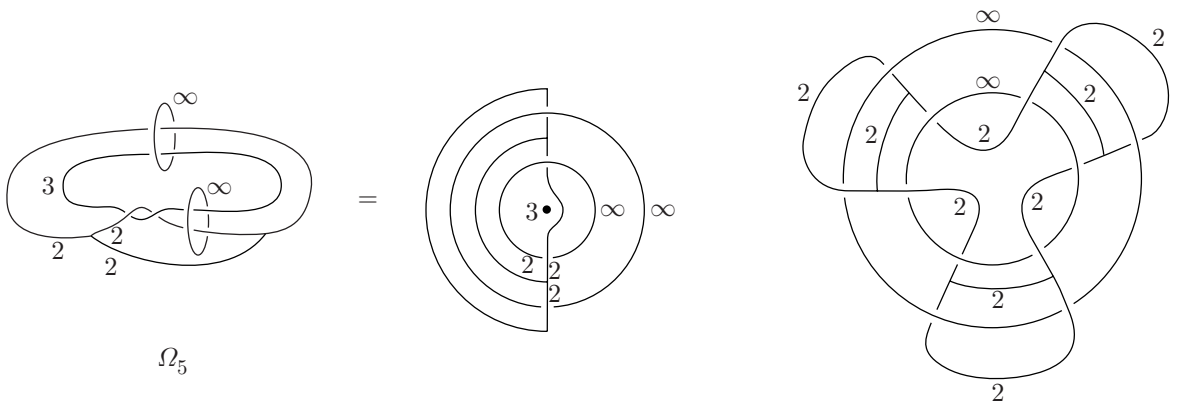

3-fold cyclic branched cover
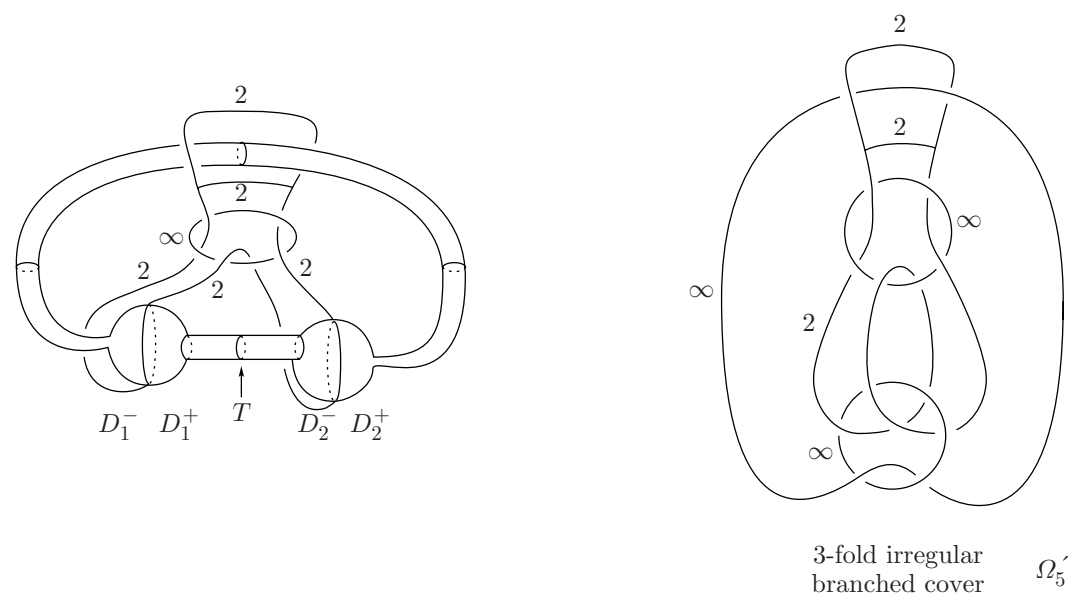

Figure 7a. Construction of $\Omega_{5}^{\prime}$

homeomorphic to $S^{3}$ with singular locus a figure eight knot of cone angle $\pi$. The construction of $X^{\prime}$ is carried out in $\S 3.4 .1$ below (see Figures 10d 10j).

The existence of $X^{\prime}$ implies that $X$ is 60 -fold orbifold covered by a nonsingular $S^{3}$, for $X^{\prime}$ is 2 -fold branch covered by the lens space $L(5,2)$ which is in turn 5 fold covered by $S^{3}$. Now, removing the link circle from $X$ and its preimages from $S^{3}$ yields a 60 -fold orbifold cover of $\Omega_{19}$ by a link complement which, we claim, corresponds to the group $\Gamma(I)$. Indeed, by construction the group $\Gamma$ of the link complement is generated by conjugates of the matrix $\left[\begin{array}{ll}1 & \omega \\ 0 & 1\end{array}\right]$ so that $\Gamma \subset \Gamma(I)$ and thus $\Gamma=\Gamma(I)$ since both are of index 60 in $\operatorname{PSL}_{2}\left(\mathcal{O}_{19}\right)$.

Actually constructing this link seemed rather difficult so we obtained the 4component link complement $\widetilde{\Omega}_{19}$ (Figure 10c) as follows. Removing the link circle from $X$ and its preimages from $X^{\prime}$ yields a 6 -fold irregular orbifold cover, $\Omega_{19}^{\prime}$, of $\Omega_{19}$ pictured in Figure 10a Explicitly, one obtains $\Omega_{19}^{\prime}$ by removing the link circle from $X$ and performing the same cutting and pasting as in the construction of $X^{\prime}$ 

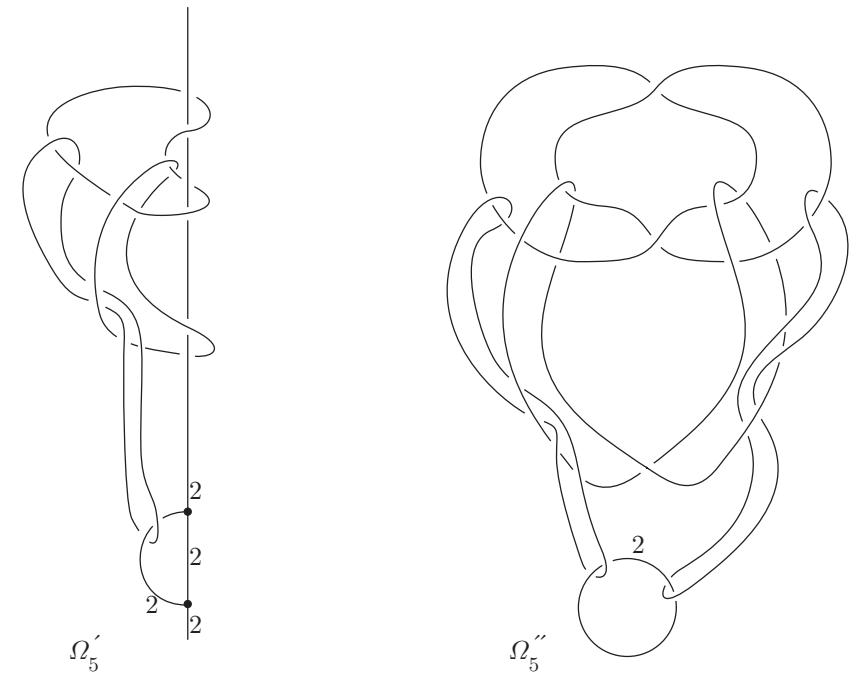

(2-fold cyclic branched cover)

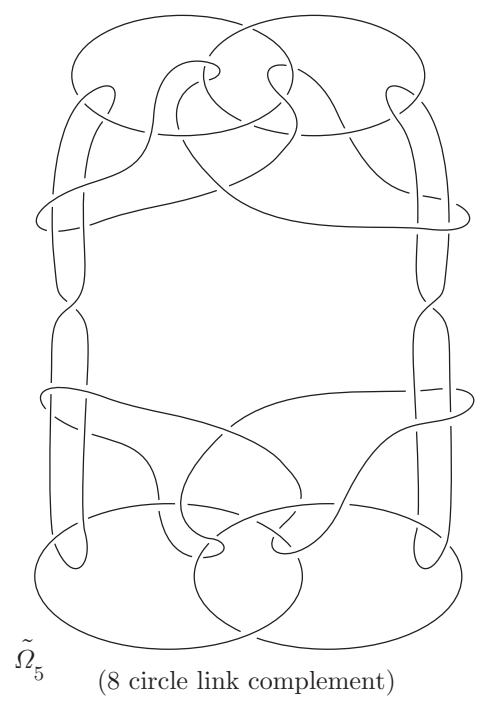

FiguRE 7b. Construction of $\widetilde{\Omega}_{5}$

given below. We omit the details. Now one easily obtains $\widetilde{\Omega}_{19}$ as illustrated in Figures $10 \mathrm{~b} 10 \mathrm{c}$; first twist in the top component of the link in $\Omega_{19}^{\prime}$ so as to unknot the singular circle, then take the appropriate 2 -fold branched cover.

3.4.1. We conclude by constructing a 6-fold irregular orbifold cover, $X^{\prime}$, of $X$ homeomorphic to $S^{3}$ with singular locus a figure eight knot of cone angle $\pi$. This is done in steps $1-5$ below (see Figures 10d, 10j).

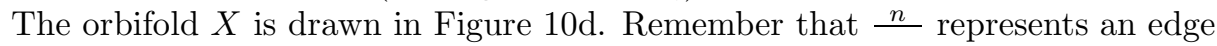
of cone angle $2 \pi / n$. We have added arrows to the edges of the singular locus to aid 


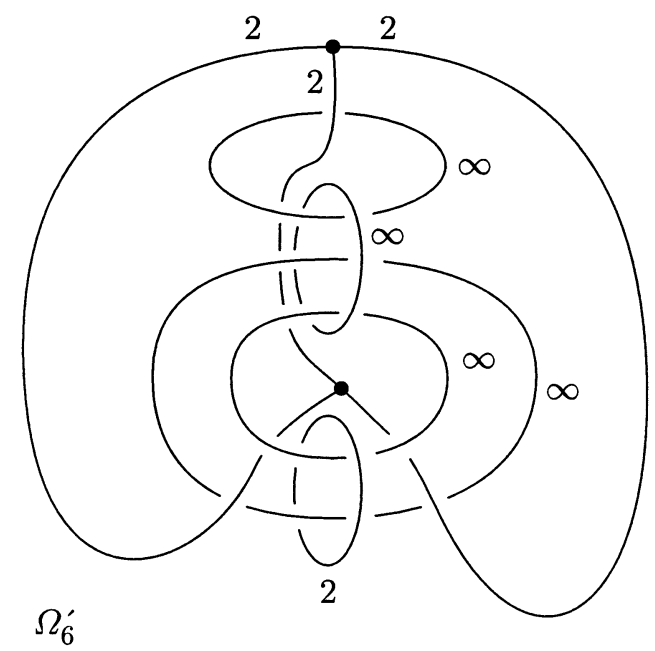

Figure 8a.

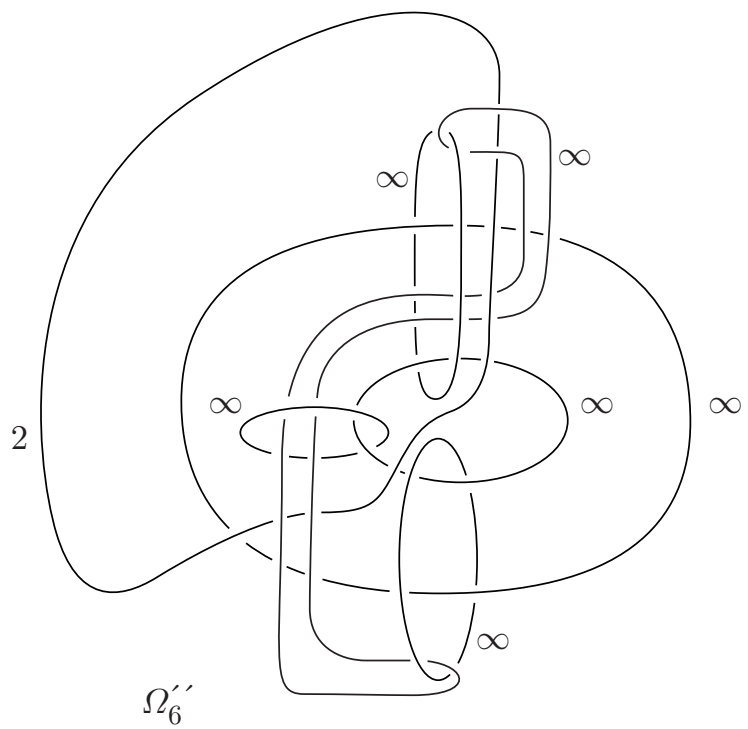

Figure $8 \mathrm{~b}$.

in following the construction. The orbifold $X^{\prime}$ is obtained by cutting and pasting together 6 copies of $X$ along disks which are bounded by loops in the singular locus of $X$.

1. Redraw $X$ as in Figure 10e In Figure 10f we have split $S^{3}$ open along a disk $D$ bounded by a loop in the singular locus of $X$. Note that the segment $\stackrel{3}{\rightarrow}$ has been split into two segments of cone angle $\pi / 3$, one on each copy of $D$.

2. Glue together two copies of $X$ along $D$ as shown in Figure $10 \mathrm{~g}$ to obtain the cone manifold $X_{2}$. Note that $X_{2}$ is not an orbifold cover of $X$, since the labels on its singular locus are not all integers. 


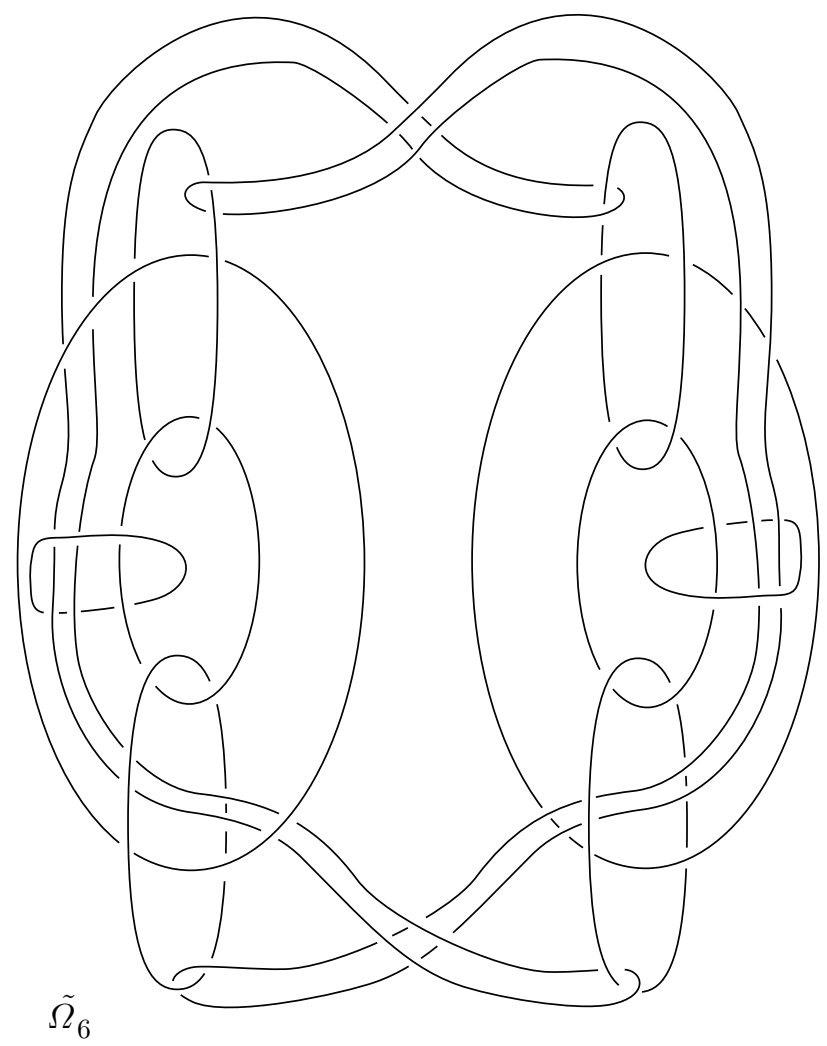

Figure 8c.

3. Glue a copy of $X$ to $X_{2}$ from the "back" of $X_{2}$ (cut and paste along disk $E$ ) to get $X_{3}$ as pictured in Figure $10 \mathrm{~h}$ Note cancellation of singular loci 3 and $3 / 2$ since $2 \pi / 3+2 \pi /(3 / 2)=2 \pi$.

4. Now glue a copy of $X$ to $X_{2}$ from the "front" (cut and paste along disk $F$ ) to get $X_{3}^{\prime}$ as pictured in Figure 10i

5. Finally, gluing $X_{3}$ to $X_{3}^{\prime}$ (along disk $G$ ) gives the desired cover $X^{\prime}$ of $X$ drawn in Figure 10j

\section{The orbifolds $\Omega_{m}$}

The orbifold $\Omega_{m}=\mathbb{H}^{3} / \mathrm{PSL}_{2}\left(\mathcal{O}_{m}\right)$ is topologically a noncompact 3-manifold with cusps of the form $T^{2} \times[0, \infty)$ for $m \neq 1,3$ (resp. $S^{2} \times[0, \infty)$ for $m=1,3$ ). In all cases, the number of cusps of $\Omega_{m}$ is equal to the class number of $\mathcal{O}_{m}$ (see [S]).

It is precisely for the 14 values of $m$ in the list $\mathcal{L}$ that $\Omega_{m}$ embeds in $S^{3}$. The orbifolds for $m=5,6,15,19,23,31,39,47,71$ are drawn in Figure 1 .

We obtained these embeddings as follows.

4.1. The cases $m=5,6,15,19,23,31$. Let $\Gamma_{m}=\operatorname{PSL}_{2}\left(\mathcal{O}_{m}\right)$ and $D_{m}$ be a Ford fundamental polyhedron for the action of $\Gamma_{m}$ on $\mathbb{H}^{3}$ (see $[\mathrm{Sw}$ for details of this construction. The domains $D_{m}, m \leq 19$, are also explicitly given). R. Riley has written a computer program (described in $[\mathrm{R} 2]$ ) that computes the Ford domains 


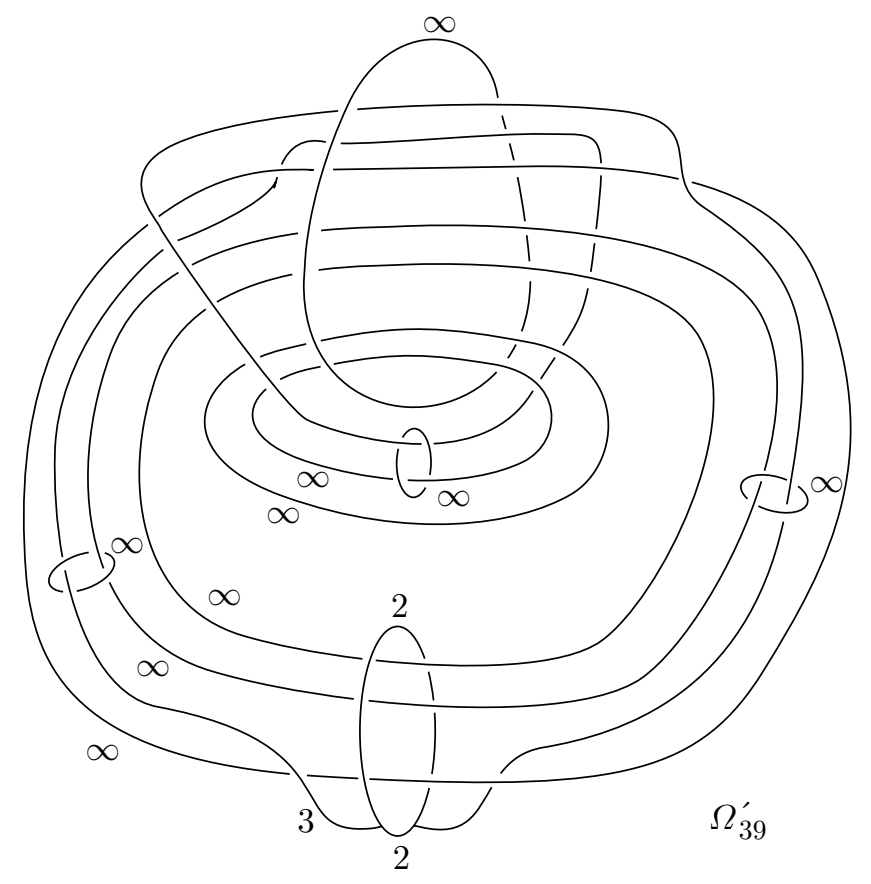

Figure 9.

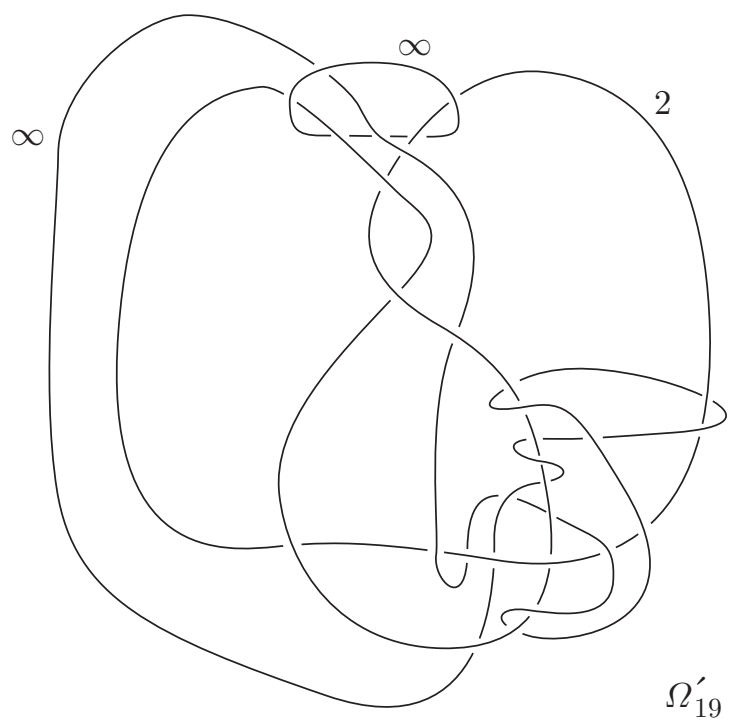

FiguRE 10a.

and face pairings for $\operatorname{PSL}_{2}\left(\mathcal{O}_{m}\right)$ and $\mathrm{PGL}_{2}\left(\mathcal{O}_{m}\right)$. Copies of the program/domains are available from him upon request.

Let $D_{m}^{*}$ be the compactification of $D_{m}$ obtained by removing a horoball neighborhood about each of the cusps. 


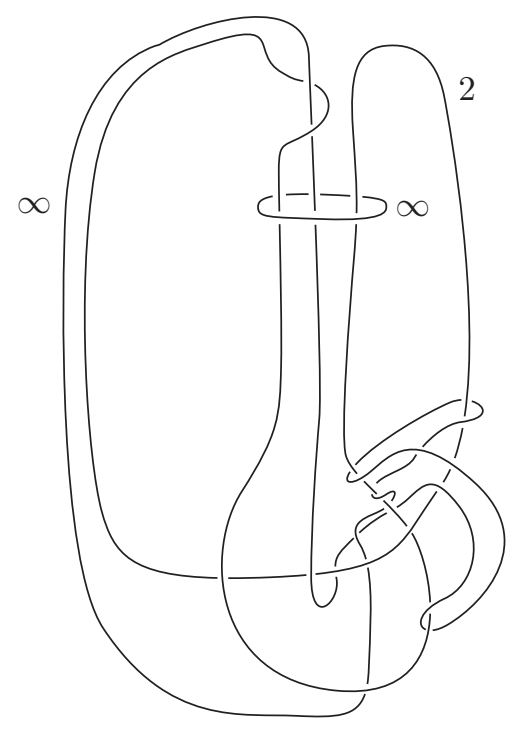

Figure 10b.

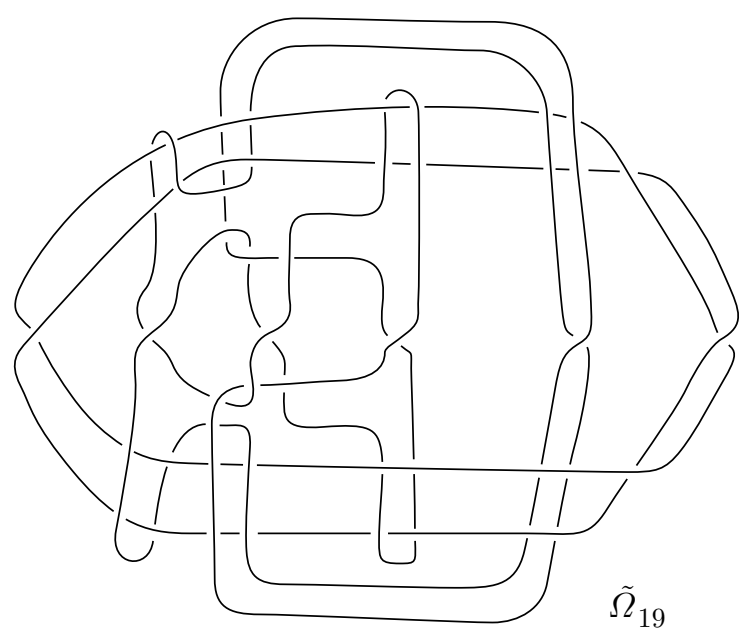

Figure 10c.

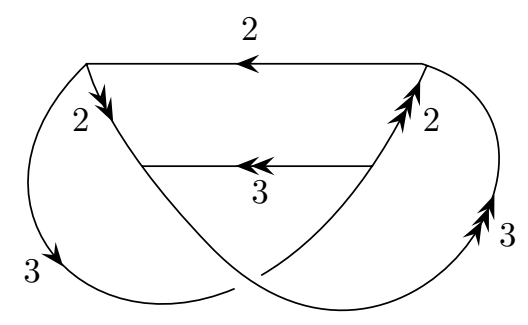

Figure 10d. 


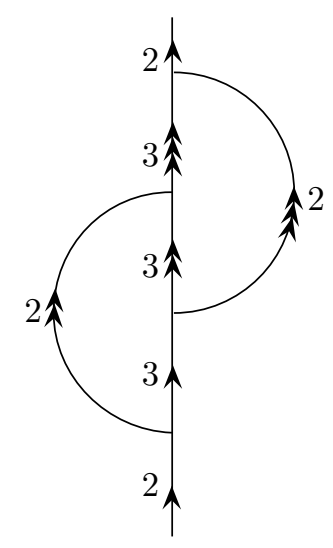

Figure 10e.

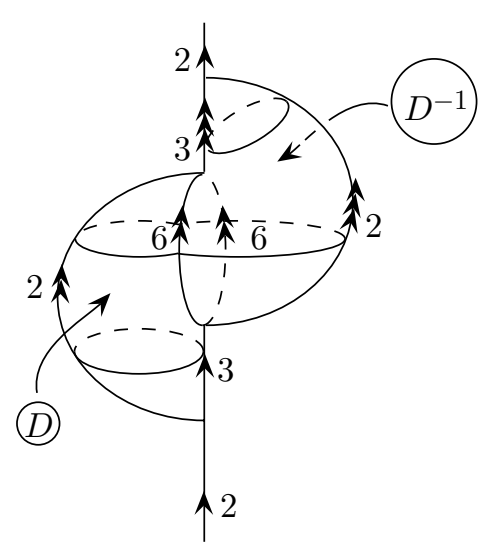

FiguRe 10f.

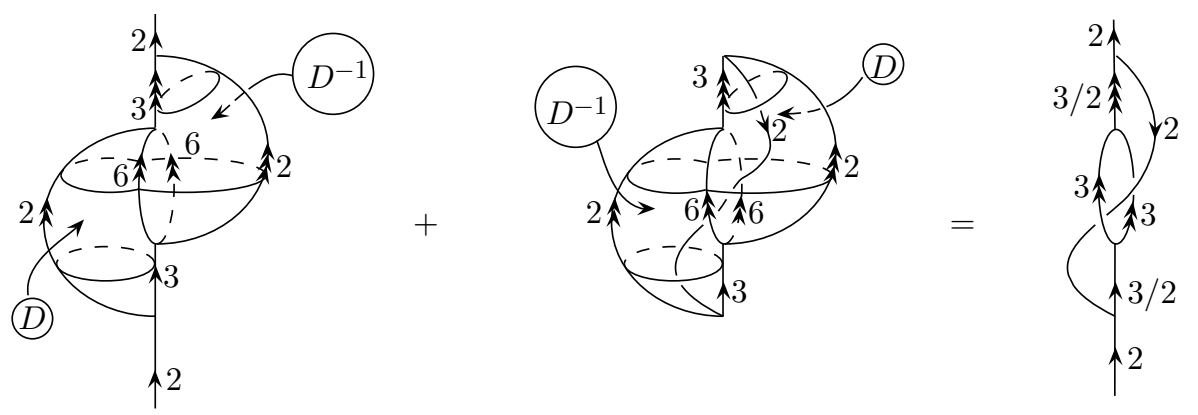

Figure 10g. Construction of $X_{2}$

For our purposes, the key facts concerning $D_{m}^{*}(m \neq 1,3)$ are:

i) $D_{m}^{*} / \Gamma_{m}$ is topologically a compact 3-manifold with interior homeomorphic to $\Omega_{m}=D_{m} / \Gamma_{m}$ and boundary a disjoint union of tori. 

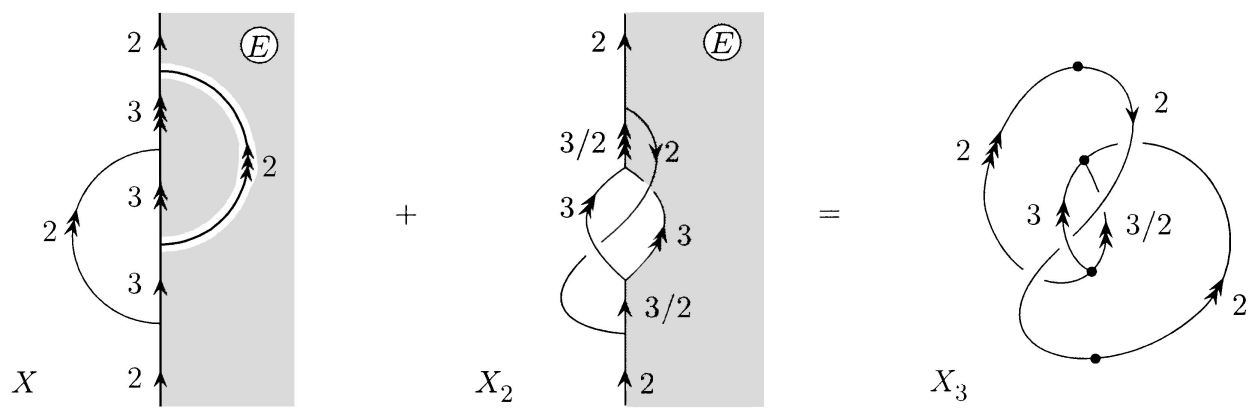

Figure 10h. Construction of $X_{3}$
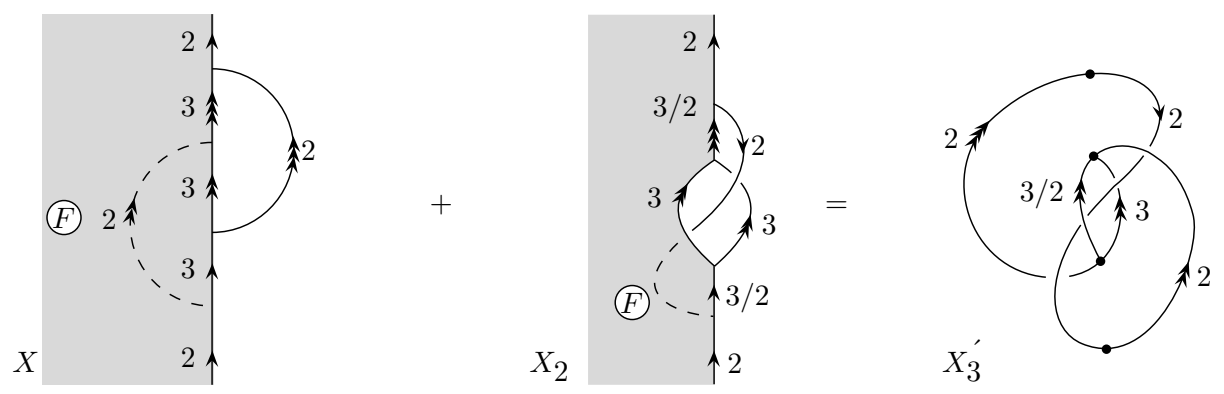

Figure 10i. Construction of $X_{3}^{\prime}$
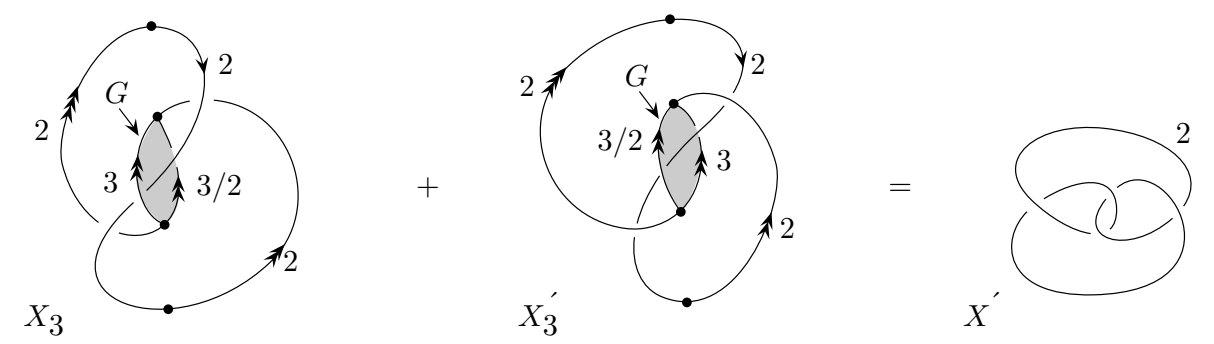

FIgURE 10 j. Construction of $X^{\prime}$

ii) Letting $\Gamma_{m}^{\infty}$ denote the parabolic subgroup of $\Gamma_{m}$ fixing $\{\infty\}$, then $D_{m}^{*} / \Gamma_{m}^{\infty}$ is homeomorphic to $T^{2} \times[0,1]$ with $T^{2} \times\{1\}$ the compactification of the cusp at $\{\infty\}$ and $T^{2} \times\{0\}$ containing the singular locus of $\Omega_{m}$ as well as the remaining unpaired faces of $D_{m}^{*}$.

Thus the first step in obtaining $\Omega_{m}$ is to embed $D_{m}^{*} / \Gamma_{m}^{\infty} \cong T^{2} \times[0,1]$ in $S^{3}=$ $\mathbb{R}^{3} \cup\{\infty\}$ so that:

1) $T^{2} \times\{1\}$ is the boundary of an open tubular neighborhood of the circle, $C$, formed by the $z$-axis of $\mathbb{R}^{3}$ and the point $\{\infty\}$.

2) The loop on $T^{2} \times\{1\}$ corresponding to the element $\left[\begin{array}{ll}1 & 1 \\ 0 & 1\end{array}\right]$ (resp. the element $\left.\left[\begin{array}{ll}1 & \omega \\ 0 & 1\end{array}\right]\right)$ of $\Gamma_{m}$ is parallel to $C$ (resp. links $C$ ). 


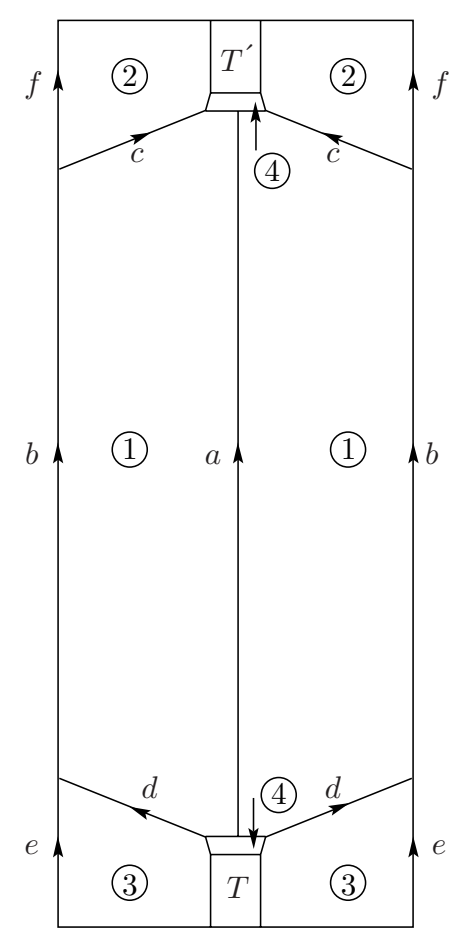

$D_{6}^{*} \quad$ (projection onto $\mathbb{C}$ with face pairings indicated)

Edges cone angle $\pi: a, e, f$

Edges cone angle $2 \pi / 3: \quad b, c, d$
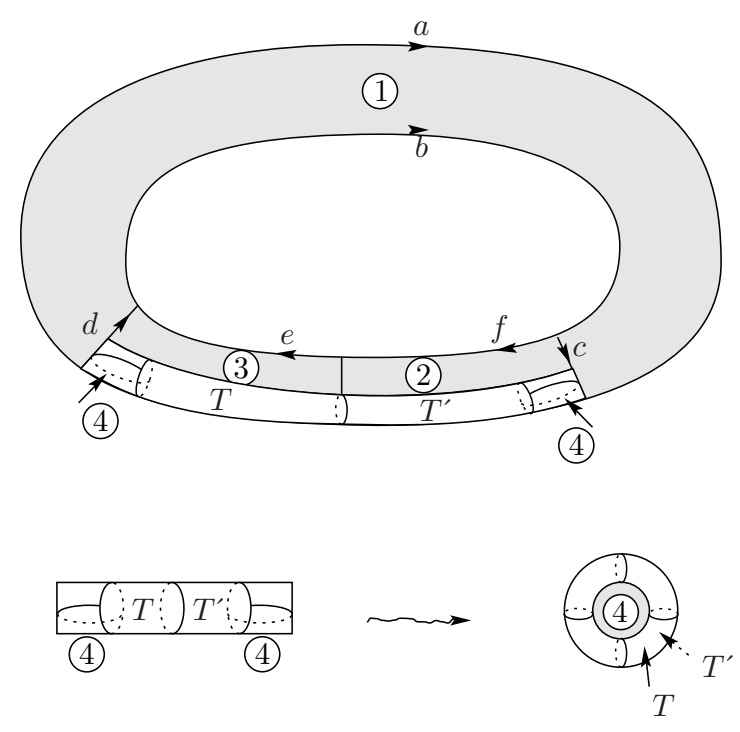

$D_{6}^{*} / \Gamma_{6}^{\infty} \quad$ with, in addition,

face pairs (1), (2), (3)

identified

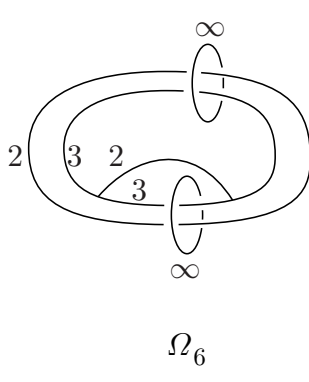

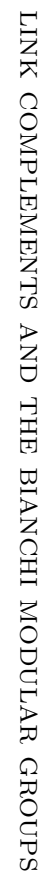

$\underset{\substack{W \\ 心}}{\stackrel{w}{W}}$

Figure 11. Embedding $\Omega_{6}$ 


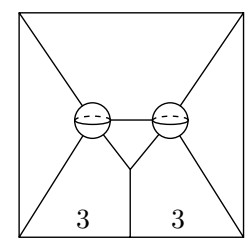

$X_{6}$ (labels $n=2$ omitted)
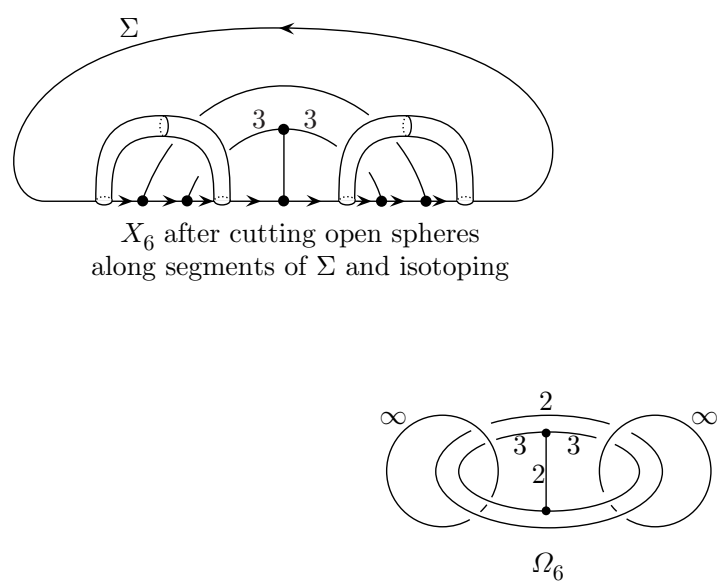

Figure 12 . Obtaining $\Omega_{6}$ from $X_{6}$

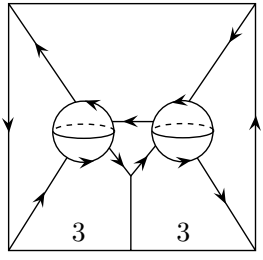

Circle $\Sigma$ : segments with arrows

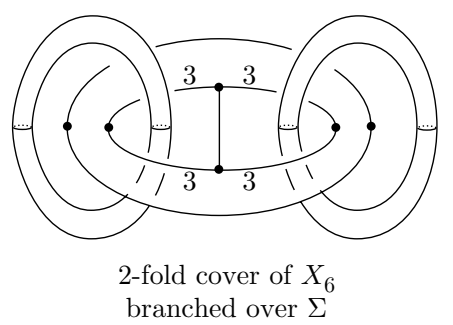

branched over $\Sigma$ 
4.3. Modification of embeddings: Twist homeomorphisms. In certain cases, we have modified our embeddings of $\Omega_{m}$ in $S^{3}$ by:

1) Twisting about unknotted link components

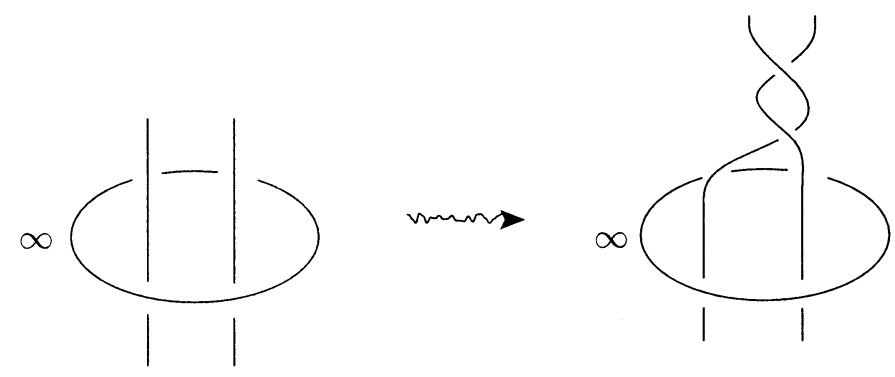

2) Twisting about parallel link components
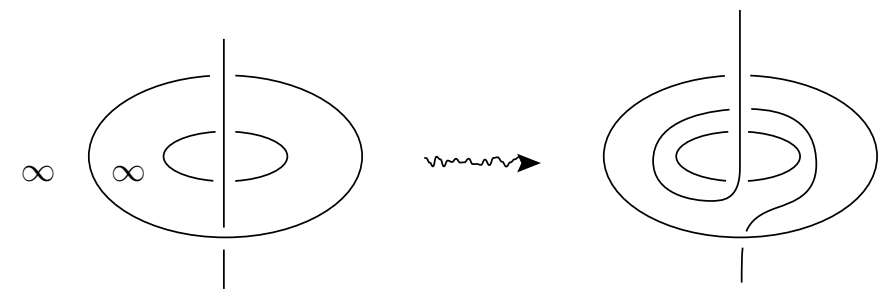

These twist homeomorphisms allow us to unknot singular circles as well as to link and unlink different components of a singular locus (see [Ro], Chapter 9, for a discussion of twist homeomorphisms).

The orbifolds $\Omega_{m}$ pictured in Figure 1 are embedded in a way that is most convenient for constructing the covers $\widetilde{\Omega}_{m}$, and hence may differ from the embeddings appearing elsewhere.

\section{Volume of $\widetilde{\Omega}_{m}$}

A nice property of the Bianchi orbifolds is the existence of a number theoretical formula, due to Humbert, for their volume (see [T], Chapter 7):

$$
\operatorname{vol}\left(\Omega_{m}\right)=\frac{(D)^{3 / 2} \zeta_{\mathbb{Q} \sqrt{-m}}(2)}{24 \zeta_{\mathbb{Q}}(2)}, \quad D=\left\{\begin{array}{l}
4 m, m \equiv 1,2(\bmod 4) \\
m, m \equiv 3 \quad(\bmod 4)
\end{array}\right.
$$

Thus the volume of $\Omega_{m}$ and hence that of $\widetilde{\Omega}_{m}$ can be computed to any degree of accuracy desired using the number theory program Pari $([\mathrm{P}])$.

As a check of our results, we used SnapPea $([\mathrm{W}])$ to analyze $\widetilde{\Omega}_{m}, \Omega_{39}^{\prime}$ and $\Omega_{71}$ in Figures 11-10 and found that their volumes agreed with those obtained from 
Humbert's formula. We give these volumes below:

$$
\begin{aligned}
\operatorname{vol}\left(\widetilde{\Omega}_{5}\right) & =50.4476311137 \ldots \\
\operatorname{vol}\left(\widetilde{\Omega}_{6}\right) & =62.1860747738 \ldots \\
\operatorname{vol}\left(\widetilde{\Omega}_{15}\right) & =18.8316833668 \ldots \\
\operatorname{vol}\left(\widetilde{\Omega}_{19}\right) & =31.8377775733 \ldots \\
\operatorname{vol}\left(\widetilde{\Omega}_{23}\right) & =38.6951532243 \ldots \\
\operatorname{vol}\left(\widetilde{\Omega}_{31}\right) & =54.0663025806 \ldots \\
\operatorname{vol}\left(\Omega_{39}^{\prime \prime}\right) & =82.787851850 \ldots
\end{aligned}
$$

( $\Omega_{39}^{\prime \prime}$ is the 2 -fold branched cover of $\Omega_{39}^{\prime}$. Its singular locus is an unknotted circle of cone angle $2 \pi / 3$ )

$$
\begin{aligned}
\operatorname{vol}\left(\widetilde{\Omega}_{47}\right) & =116.606541915 \ldots \\
\operatorname{vol}\left(\Omega_{71}\right) & =37.533306130 \ldots
\end{aligned}
$$

\section{ACKNowledGMEnts}

I thank F. Bonahon for helpful correspondence which led me to consider the orbifolds $\Omega_{m}$. I also thank A. Hatcher for providing me with his $\mathrm{PGL}_{2}\left(\mathcal{O}_{m}\right)$ orbifold drawings which greatly facilitated my treatment of the cases $m=39,47,71$.

\section{REFERENCES}

[B] M. Baker, Link complements and integer rings of class number greater than one, Topology '90 (Proceedings of the research semester in Low Dimensional Topology at Ohio State University), Walter de Gruyter, Berlin, New York (1992), 55-59. MR 93k:57007

[G] GAP (Groups, algorithms, and programming). Available from: http://www.gap.dcs.stand.ac.uk/ gap/.

[H1] A. Hatcher, Hyperbolic structures of arithmetic type on some link complements, J. London Math. Soc. (2), 27 (1983), 345-355. MR 84m:57005

[H2] A Hatcher, Bianchi orbifolds of small discriminant, preprint (available from author's web page).

[P] Pari, Available from Université de Bordeaux: http://www.math.u-bordeaux.fr/A2X.

[R1] R. Riley, A quadratic parabolic group, Math. Proc. Cambridge Philos. Soc. 77 (1975), 281288. MR 54:542

[R2] R. Riley, Applications of a computer implementation of Poincaré's theorem on fundamental polyhedra, Math. Comp. 40 (1983), 607-632. MR 85b:20064

[Ro] D. Rolfsen, Knots and Links, Publish or Perish, Inc., Berkeley, (1976). MR 58:24236, corrected reprint MR 95c:57018

[S] J.-P. Serre, Le problème des groupes de congruence pour $\mathrm{SL}_{2}$, Ann. of Math. 92 (1972), 489-527. MR 42:7671

[Sw] R. Swan, Generators and relations for certain special linear groups, Adv. in Math. 6 (1971), 1-77. MR 44:1741

[T] W. Thurston, The geometry and topology of 3-manifolds, Mimeographed notes, Princeton University, 1978.

[V] K. Vogtmann, Rational homology of Bianchi groups, Math. Ann. 272 (1985), 399-419. MR 87a:22025

[W] J. Weeks, SnapPea 2.4 PPC. Available from: weeks@geom.umn.edu.

[Wi] N. Wielenberg, The structure of certain subgroups of the Picard group, Math. Proc. Cambridge Philos. Soc. 84 (1978), 427-436. MR 80b:57010

IRMAR, Université de Rennes 1, 35042 Rennes Cedex, France 